\title{
BMJ Open Independent and joint effects of sedentary time and cardiorespiratory fitness on all-cause mortality: the Cooper Center Longitudinal Study
}

\author{
Kerem Shuval, ${ }^{1}$ Carrie E Finley, ${ }^{2}$ Carolyn E Barlow, ${ }^{2}$ Binh T Nguyen, ${ }^{1}$ \\ Valentine $\mathrm{Y}$ Njike, ${ }^{3}$ Kelley Pettee Gabriel $^{4}$
}

To cite: Shuval K, Finley CE, Barlow CE, et al. Independent and joint effects of sedentary time and cardiorespiratory fitness on all-cause mortality: the Cooper Center Longitudinal Study. BMJ Open 2015;5:e008956. doi:10.1136/bmjopen-2015008956

- Prepublication history for this paper is available online. To view these files please visit the journal online (http://dx.doi.org/10.1136/ bmjopen-2015-008956).

Received 1 June 2015 Revised 10 September 2015 Accepted 6 October 2015

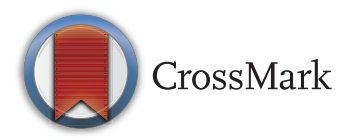

${ }^{1}$ Intramural Research Department, American Cancer Society, Atlanta, Georgia, USA

${ }^{2}$ The Cooper Institute, Dallas, Texas, USA

${ }^{3}$ Yale University Prevention Research Center, Griffin Hospital, Derby, Connecticut, USA

${ }^{4}$ Division of Epidemiology, Human Genetics and Environmental Sciences, University of Texas, School of Public Health, Austin, Texas, USA

Correspondence to Dr Kerem Shuval; kerem.shuval@cancer.org

\section{ABSTRACT}

Objectives: To examine the independent and joint effects of sedentary time and cardiorespiratory fitness (fitness) on all-cause mortality.

Design, setting, participants: A prospective study of 3141 Cooper Center Longitudinal Study participants. Participants provided information on television (TV) viewing and car time in 1982 and completed a maximal exercise test during a 1-year time frame; they were then followed until mortality or through 2010. TV viewing, car time, total sedentary time and fitness were the primary exposures and all-cause mortality was the outcome. The relationship between the exposures and outcome was examined utilising Cox proportional hazard models.

Results: A total of 581 deaths occurred over a median follow-up period of 28.7 years $(\mathrm{SD}=4.4)$. At baseline, participants' mean age was 45.0 years (SD=9.6), 86.5\% were men and their mean body mass index was 24.6 $(S D=3.0)$. Multivariable analyses revealed a significant linear relationship between increased fitness and lower mortality risk, even while adjusting for total sedentary time and covariates $(p=0.02)$. The effects of total sedentary time on increased mortality risk did not quite reach statistical significance once fitness and covariates were adjusted for $(p=0.05)$. When examining this relationship categorically, in comparison to the reference category ( $\leq 10 \mathrm{~h} /$ week), being sedentary for $\geq 23 \mathrm{~h}$ weekly increased mortality risk by $29 \%$ without controlling for fitness (HR=1.29, 95\% Cl 1.03 to 1.63); however, once fitness and covariates were taken into account this relationship did not reach statistical significance (HR=1.20, 95\% Cl 0.95 to 1.51). Moreover, spending $>10 \mathrm{~h}$ in the car weekly significantly increased mortality risk by $27 \%$ in the fully adjusted model. The association between TV viewing and mortality was not significant.

Conclusions: The relationship between total sedentary time and higher mortality risk is less pronounced when fitness is taken into account. Increased car time, but not TV viewing, is significantly related to higher mortality risk, even when taking fitness into account, in this cohort.

\section{BACKGROUND}

Sedentary behaviour and health has emerged as a new area of scientific investigation, based
Strengths and limitations of this study

The first study, to our knowledge, to examine the effects of sedentary behaviour on mortality, while taking cardiorespiratory fitness into account.

- Cardiorespiratory fitness was assessed objectively via maximal exercise testing; however, sedentary behaviour was based on self-report.

- While the study sample consists of participants with extensive clinical and behavioural information with a long duration of follow-up, the sample was drawn from a single preventive medicine clinic.

on accumulating studies linking prolonged sitting to morbidity and mortality. ${ }^{1}$ In the USA, adults spend close to $8 \mathrm{~h}$ daily in sedentary behaviours, defined as low energy expenditure activities (1.0-1.5 metabolic equivalents (METs)) in a sitting or reclining posture. ${ }^{23}$ These prolonged hours of sedentary time have been found to be related to cardiometabolic risk (primarily in crosssectional studies), ${ }^{4-7}$ and premature death from all causes and from cardiovascular diseases in prospective studies. ${ }^{8-10}$ For example, a review by Ford and Caspersen ${ }^{10}$ observed a $17 \%$ increased risk for cardiovascular events (fatal and non-fatal) per $2 \mathrm{~h} /$ day increments of television (TV) viewing, and $5 \%$ more cardiovascular events per $2 \mathrm{~h}$ increases in sitting time. Additionally, recent meta-analyses by Chau et $a \ell^{\dagger}$ and Biswas $e t a l^{11}$ found a $34 \%$ and $24 \%$ higher risk (respectively) for all-cause mortality for prolonged sedentary time, even after adjusting for physical activity.

These studies, however, have predominately taken into account self-reported physical activity (which is prone to recall bias), and have yet to control for cardiorespiratory fitness (fitness). Fitness, an objective and physiological consequence of habitual 
physical activity (also influenced by genetics) is an indicator of overall cardiovascular health. ${ }^{12-14}$ Observational evidence has found that low fitness levels accounted for $\sim 16 \%$ of deaths in a large cohort of over 40000 individuals, ${ }^{15}$ yet to date, studies have not accounted for fitness when examining the effects of sedentary behaviour on mortality. Hence, we attempt to bridge this gap by examining whether sedentary behaviour is associated with increased mortality risk, while considering the potential mitigating effects of fitness. Specifically, we examine the independent and joint effects of sedentary time and fitness on all-cause mortality among participants of the Cooper Center Longitudinal Study (CCLS).

\section{METHODS}

\section{Participants and design}

The CCLS, described elsewhere,${ }^{16}$ is an observational study of patients who self-referred or were referred by their employer or physician to the Cooper Clinic (Dallas, Texas) for preventive medical examinations. ${ }^{17}$ In general, the CCLS aims to examine the effects of fitness on chronic disease morbidity and mortality. ${ }^{18}$ The CCLS receives annual approval from the Cooper Institute Institutional Review Board and the present investigation received approval from the Committee for the Protection of Human Subjects at the University of Texas Health Science Center at Houston. In the current study, we assessed the effects of sedentary behaviour and fitness on all-cause mortality among adults ( $\geq 20$ years) who: (1) completed a 1982 survey including questions pertaining to sedentary behaviour; and (2) came for a preventive medical visit which included a fitness test and a thorough medical history questionnaire at the Cooper Clinic within a 1 year time frame. ${ }^{12}$ Of 3676 participants meeting these criteria with pertinent data on the study measures, 329 were excluded due to incomplete fitness testing, abnormal exercise ECG, less than 1 year of follow-up and underweight status. Additionally, 206 participants were excluded based on personal history of myocardial infarction, stroke or cancer. These exclusion criteria resulted in an analytic sample of 2716 men and 425 women (total $n=3141$ ) with complete data on the primary exposures (sedentary behaviour and fitness), and the outcome (all-cause mortality). Owing to the small number of women in the sample and the lack of a significant interaction effect between gender and the exposures $(p>0.10)$ in relation to mortality, gender was adjusted for in multivariable analyses rather than performing stratified analysis.

\section{Measures}

Exposures (sedentary behaviour and cardiorespiratory fitness)

Sedentary behaviour was assessed at baseline via reported time spent viewing TV and commuting in a car, as indicated in a 1982 survey. ${ }^{12}{ }^{19}$ Specifically, participants were asked the following two questions pertaining to their sedentary behaviour: (1) 'How much time do you spend riding in a car each week?__hours per week'; and (2) 'How much time do you spend watching TV each week?_hours per week'. For analysis, the hours of car driving and TV viewing per week were considered separate exposure variables. The combined amount (hours per week) of sedentary time (ie, the sum of TV viewing and car commuting time) was regarded as an additional exposure variable. These exposure variables were each categorised into sample-specific quartiles. Quartile cut-points for the combined sedentary time are: $11,16,23 \mathrm{~h}$ /week; the quartile cut-points of TV viewing and car time appear in table 1. Fitness was assessed via maximal exercise testing on a treadmill adhering to the modified Balke protocol. In this protocol, described elsewhere, ${ }^{16}$ the treadmill speed and incline are increased gradually up to 25 min or until volitional exhaustion. ${ }^{20}$ From the final treadmill speed and grade maximal METs $(1 \mathrm{MET}=3.5 \mathrm{~mL} \mathrm{O}$ uptake $\times \mathrm{kg} /$ body mass $/ \mathrm{min})$ were determined, which have been highly correlated $(r>0.90)$ with maximal oxygen uptake. ${ }^{21} 22$ Fitness METs of the analytic sample were categorised into age (20-39, $40-49, \quad 50-59$ and $\geq 60$ years) specific tertiles (low, medium, high) for each gender separately. ${ }^{23}$

\section{Outcome (all-cause mortality)}

Participants were followed for mortality from all causes from baseline to either the date of death or through 31 December 2010 in order to determine vital status. The National Death Index (NDI) was the primary source of mortality information. ${ }^{18}$ The NDI has been found to have $100 \%$ specificity and $96 \%$ sensitivity in ascertaining mortality among the general population. ${ }^{24} 25$

\section{Covariates}

Covariates include age, gender, current smoking, alcohol intake, personal history of hypertension and diabetes, family history of cardiovascular disease, leisuretime physical activity, body mass index (BMI), blood pressure, total cholesterol, low-density lipoprotein cholesterol, high-density lipoprotein (HDL)-cholesterol, and fasting glucose. Participants' age, gender, current smoking status and alcohol intake (drinks per week) were based on responses to a medical history questionnaire. Alcohol intake was categorised for analyses into: non-drinkers, (2) light drinkers ( $\leq 3$ drinks a week for women and men), moderate drinkers ( $>3$ to 7 drinks a week for women or $>3$ to 14 drinks a week for men) and heavy drinkers ( $>7$ drinks per week for women or $>14$ drinks/week for men). ${ }^{20}{ }^{26}$ Leisure-time physical activity was based on survey questions pertaining to the frequency and the amount of time spent in the following activities: running, treadmill, swimming, stationary cycling, bicycling, elliptical, aerobic dance, racket sports, vigorous sports and other activity. ${ }^{27}$ MET values for each activity were based on the physical activity compendium and multiplied by the frequency and intensity of activity performed resulting in MET $\mathrm{min} /$ week $^{28}$ The sum of 
Table 1 Baseline characteristics of participants by vital status, the Cooper Center Longitudinal Study

\begin{tabular}{|c|c|c|c|c|}
\hline & All & Survivors & Decedents & p Value* \\
\hline $\mathrm{N}$ & 3141 & 2560 & 581 & \\
\hline Men, n (\%) & $2716(86.5)$ & $2206(86.2)$ & $510(87.8)$ & 0.306 \\
\hline Women, n (\%) & 425 (13.5) & $354(13.8)$ & 71 (12.2) & \\
\hline Follow-up (years) & $27.3(4.4)$ & $28.9(0.7)$ & $20.4(6.4)$ & $<0.001$ \\
\hline Age (years) & $45.0(9.6)$ & $43.1(8.6)$ & $53.1(9.7)$ & $<0.001$ \\
\hline Resting systolic blood pressure (mm Hg) & $116.9(12.7)$ & $115.8(12.1)$ & $121.7(14.1)$ & $<0.001$ \\
\hline Resting diastolic blood pressure $(\mathrm{mm} \mathrm{Hg})$ & $78.9(8.7)$ & $78.3(8.4)$ & $81.4(9.5)$ & $<0.001$ \\
\hline Total cholesterol (mg/dL) & $204.8(34.4)$ & $203.4(34.1)$ & $211.1(35.2)$ & $<0.001$ \\
\hline LDL-C (mg/dL) & $134.3(31.3)$ & $133.3(31.1)$ & $138.8(31.8)$ & $<0.001$ \\
\hline HDL-C (mg/dL) & $48.0(11.8)$ & $48.0(11.6)$ & $47.8(12.4)$ & 0.683 \\
\hline Triglycerides (mg/dL) & $112.8(61.6)$ & $110.6(61.0)$ & $122.6(63.7)$ & $<0.001$ \\
\hline Glucose (mg/dL) & $96.2(13.3)$ & $95.3(11.7)$ & $100.3(18.5)$ & $<0.001$ \\
\hline Body mass index $\left(\mathrm{kg} / \mathrm{cm}^{2}\right)$ & $24.6(3.0)$ & $24.5(3.0)$ & $25.2(3.3)$ & $<0.001$ \\
\hline \multicolumn{5}{|l|}{ Physical activity guidelines $\dagger$} \\
\hline Not meeting guidelines & $1798(57.2)$ & $1460(57.0)$ & $338(58.2)$ & \multirow[t]{3}{*}{0.609} \\
\hline Meeting guidelines & $571(18.2)$ & $467(18.2)$ & $104(17.9)$ & \\
\hline Exceeding guidelines & $772(24.6)$ & $633(24.7)$ & 139 (23.9) & \\
\hline \multicolumn{5}{|l|}{ Cardiorespiratory fitness $\ddagger$, n (\%) } \\
\hline Low & 1105 (35.2) & $843(32.9)$ & $262(45.1)$ & \multirow[t]{3}{*}{$<0.001$} \\
\hline Middle & 1025 (32.6) & $854(33.4)$ & $171(29.4)$ & \\
\hline High & 1011 (32.2) & 863 (33.7) & $148(25.5)$ & \\
\hline \multicolumn{5}{|l|}{ Car time§, n (\%) } \\
\hline Q1 & 925 (29.4) & $746(29.1)$ & $179(30.8)$ & \multirow[t]{4}{*}{0.8904} \\
\hline Q2 & $848(27.0)$ & $695(27.1)$ & $153(26.3)$ & \\
\hline Q3 & $637(20.3)$ & $534(20.9)$ & $103(17.4)$ & \\
\hline Q4 & 731 (23.3) & 585 (22.9) & $146(25.1)$ & \\
\hline \multicolumn{5}{|l|}{ TV viewing§, $n(\%)$} \\
\hline Q1 & $793(25.2)$ & $668(26.1)$ & $125(21.5)$ & \multirow[t]{4}{*}{0.0034} \\
\hline Q2 & $837(26.6)$ & $697(27.2)$ & $140(24.1)$ & \\
\hline Q3 & $812(25.9)$ & $636(24.8)$ & $176(30.3)$ & \\
\hline Q4 & 699 (22.3) & $559(21.8)$ & $140(24.1)$ & \\
\hline \multicolumn{5}{|l|}{ Total sedentary time§, $\mathrm{n}(\%)$} \\
\hline Q1 & $895(28.5)$ & $749(29.3)$ & $146(25.1)$ & \multirow[t]{4}{*}{0.0081} \\
\hline Q2 & $687(21.9)$ & 571 (22.3) & $116(20.0)$ & \\
\hline Q3 & $845(26.9)$ & $673(26.3)$ & $172(29.6)$ & \\
\hline Q4 & $714(22.7)$ & $567(22.2)$ & $147(25.3)$ & \\
\hline \multicolumn{5}{|l|}{ Alcohol intakeq } \\
\hline Non-drinkers & $722(23.0)$ & $588(23.0)$ & $134(23.1)$ & \multirow[t]{4}{*}{0.3064} \\
\hline Light drinkers & $426(13.6)$ & $360(14.1)$ & $66(11.4)$ & \\
\hline Moderate drinkers & $1424(45.3)$ & $1162(45.4)$ & $262(45.1)$ & \\
\hline Heavy drinkers & $540(17.2)$ & $429(16.8)$ & $111(19.1)$ & \\
\hline Current smoker, n (\%) & $452(14.4)$ & 350 (13.7) & $102(17.6)$ & 0.003 \\
\hline Personal history of hypertension, $\mathrm{n}(\%)$ & $520(16.6)$ & $368(14.4)$ & $152(26.2)$ & $<0.001$ \\
\hline Personal history of diabetes, $\mathrm{n}(\%)$ & $60(1.9)$ & $39(1.5)$ & $21(3.6)$ & $<0.001$ \\
\hline Family history of CVD n (\%) & $451(14.4)$ & $384(15.0)$ & $67(11.5)$ & 0.031 \\
\hline
\end{tabular}

Values are mean (SD) unless otherwise indicated.

*Wald trend test $p$ values for continuous variables; Jonckeheere-Terpstra trend test $p$ values for categorical variables.

†Physical activity was based on self-reported type, time and intensity of activity which were converted into MET minutes per week. METs were then categorised into: (1) not meeting physical activity guidelines ( $<500 \mathrm{MET} \mathrm{mi/week);} \mathrm{meeting} \mathrm{physical} \mathrm{activity} \mathrm{guidelines} \mathrm{(500-1000}$ $\mathrm{MET} \mathrm{min} /$ week); and (3) exceeding physical activity guidelines (>1000 MET min/week).

‡Cardiorespiratory fitness was categorised into age (20-39, 40-49, 50-59 and $\geq 60$ years) and gender-specific tertiles based on the distribution of the sample.

$\S$ Total sedentary time (ie, the sum of reported TV viewing and car time) was categorised into sample-specific quartiles (Q): Q1 (0-10 h/week), Q2 (11-15 h/week), Q3 (16-22 h/week) and Q4 ( $\geq 23 \mathrm{~h} /$ week). Quartiles of car time: (Q): Q1 (0-4 h/week), Q2 (5-7 h/week), Q3 (8-10 h/ week) and Q4 ( $\geq 11 \mathrm{~h} /$ week). Quartiles of TV viewing: (Q): Q1 (0-3 h/week), Q2 (4-7 h/week), Q3 (8-12 h/week) and Q4 ( $\geq 13 \mathrm{~h} /$ week). ๆNon-drinker: 0 drinks per week; light drinker $\leq 3$ drinks per week; moderate drinker: $>3-7$ drinks a week for women and $>3-14$ drinks per week for men; heavy drinker $>7$ drinks per week for women and $>14$ drinks/week for men. A total of 29 participants had missing values for alcohol intake and thus a 'missing' category was utilised in multivariable analysis.

CVD, cardiovascular disease; HDL-C, high-density lipoprotein cholesterol; LDL-C, low-density lipoprotein cholesterol; MET, metabolic equivalent; Q: quartile; TV, television. 
the MET values from all activities was subsequently grouped into the following three categories based on the Health and Human Services Physical Activity Guidelines: (1) not meeting guidelines $(<500$ MET $\mathrm{min} /$ week); (2) meeting guidelines (500-1000 MET min/week); and (3) exceeding guidelines (>1000 MET min/week). ${ }^{29}$ In this study, meeting physical activity guidelines was significantly associated with cardiorespiratory fitness levels (Spearman $\mathrm{r}=0.46, \mathrm{p}<0.001$ ). In addition, personal and family history of disease was based on self-report on the medical history questionnaire. ${ }^{30} \mathrm{BMI}$ and clinical indicators were determined during the clinical examination. Specifically, BMI was computed from height and weight using the standard formula $\left(\mathrm{kg} / \mathrm{m}^{2}\right)$, resting blood pressure was measured with a calibrated sphygmomanometer, and serum samples were analysed for glucose and lipids adhering to standard procedures after a $12 \mathrm{~h}$ fast. ${ }^{18}$

\section{Statistical analysis}

Descriptive characteristics were computed for the entire sample and by vital status. The association between sedentary behaviour and all-cause mortality was determined using Cox proportional hazard models to estimate the HR and $95 \%$ CI. These models passed the proportional hazards assumption test adhering to the methodology suggested by Lin et al, ${ }^{31}$ which is based on cumulative sums of Martingale residuals. A total of four regression models were computed adjusting for the following covariates: model 1-age and gender; model 2-age, gender, current smoking (dichotomous), alcohol intake (categorical), personal history of hypertension (dichotomous), personal history of diabetes (dichotomous) and family history of cardiovascular disease (dichotomous); model 3-variables in model 2 along with fitness or total sedentary time (both categorical); and model 4-variables in model 3 as well as BMI, glucose, systolic blood pressure, total cholesterol (all continuous) and self-reported physical activity (categorical). ${ }^{18} 29$ We then reanalysed models 1 through 4 , replacing total sedentary time with either car time (categorical) or TV viewing (categorical) as the exposure of interest. Furthermore, we examined the joint effects of total sedentary time, car time and TV viewing coupled with fitness on mortality risk, while adjusting for the other covariates in model 4 . For the joint effects models, we collapsed fitness into two categories: low fitness and middle/ high fitness. Multiplicative interactions were assessed by including their cross-product in the statistical model. For all analyses, $p$ values were two sided with an $\alpha$ of $<0.05$ considered statistically significant; SAS V.9.4 (SAS Institute, Inc, Cary, North Carolina, USA) was utilised in analyses.

\section{RESULTS}

A total of 581 deaths occurred over a median follow-up period of 28.7 years $(\mathrm{SD}=4.4)$. At baseline, participants' mean age was 45.0 years $(\mathrm{SD}=9.6), 14.4 \%$ were current smokers and participants consumed a median of five alcoholic beverages per week. In addition, participants were of normal weight (mean $\mathrm{BMI}=24.6, \mathrm{SD}=3.0$ ), had an average fitness level of 12.1 METs $(\mathrm{SD}=2.4)$ and spent $17.0 \mathrm{~h} /$ week $(\mathrm{SD}=10.1)$ in total sedentary time (ie, time spent in the car and watching TV). Participants' baseline characteristics are described by vital status in table 1 .

The association between sedentary behaviours and allcause mortality is depicted in table 2. Specifically, a significant linear relationship was found between higher total sedentary time and increased mortality risk in three of the four multivariable models (linear trend $\mathrm{p}<0.05$ for models 1-3), with the fully adjusted model (including fitness, physical activity and clinical variables) not quite reaching statistical significance (linear trend $\mathrm{p}=0.05$ ). When examining this relationship categorically, being sedentary for $\geq 23 \mathrm{~h}$ weekly was significantly related to a $34 \%$ increase in mortality risk $(\mathrm{HR}=1.34$, 95\% CI 1.06 to 1.68 ) without adjusting for fitness, in comparison to the reference group $(\leq 10 \mathrm{~h}$ of sedentary time weekly). However, once fitness was included in the model (model 3) the 22\% higher mortality risk did not reach statistical significance (HR=1.22, 95\% CI 0.97 to 1.54). Moreover, in the fully adjusted model (model 4 which additionally controlled for fitness, physical activity, BMI, cholesterol, blood pressure and glucose) the $20 \%$ higher mortality risk similarly did not reach statistical significance ( $\mathrm{HR}=1.20,95 \%$ CI 0.95 to 1.51$)$. When examining the relationship between each sedentary behaviour (car time or TV viewing) and mortality, the associations differed markedly (table 2). Specifically, more time spent in a car per week was significantly associated with a higher risk for all-cause mortality in all multivariable models (linear trend $\mathrm{p}<0.05$ in all models). While the addition of fitness into the models reduced the risk for mortality, the associations still remained significant in the models. Thus, spending more than $10 \mathrm{~h}$ in the car per week increased the risk for all-cause mortality by $27 \%$ in the fully adjusted model (HR=1.27, 95\% CI 1.01 to 1.59). In comparison, the association between TV viewing and mortality was not significant in all the models (table 2). In addition, table 2 also presents the relationship between fitness and mortality while taking into account confounders. All multivariable models exhibited significant dose-response effects for increased fitness and reduced mortality risk, including models adjusting for sedentary behaviour (linear trend $\mathrm{p}<0.05$ for all). For example, in the fully adjusted model (model 4), while middle levels of fitness were associated with a $20 \%$ reduced mortality risk, high fitness levels were related to a $24 \%$ lower mortality risk in comparison to the reference group of low fitness (middle fitness: $\mathrm{HR}=0.80,95 \%$ CI 0.65 to 0.99 ; high fitness: $\mathrm{HR}=0.76$, $95 \%$ CI 0.59 to 0.97 ).

When examining the joint effects of fitness and combined sedentary behaviour on mortality, we found that in comparison to the 'high-risk' reference group (low fitness/fourth quartile of combined sedentary time) participants who were in the middle/high fitness category 
Table 2 Association between sedentary time ${ }^{*}$, cardiorespiratory fitness $\nmid$ and all-cause mortality: multivariable modelsł

\begin{tabular}{|c|c|c|c|c|c|c|}
\hline All-cause mortality & $\mathbf{n}$ & Cases & $\begin{array}{l}\text { Model } 1 \\
\text { HR (95\% Cl)§ }\end{array}$ & $\begin{array}{l}\text { Model } 2 \\
\text { HR (95\% Cl)介 }\end{array}$ & $\begin{array}{l}\text { Model } 3 \\
\text { HR }(95 \% \mathrm{Cl})^{\star \star}\end{array}$ & $\begin{array}{l}\text { Model } 4 \\
\text { HR (95\% Cl)t† }\end{array}$ \\
\hline \multicolumn{7}{|l|}{ Car time* } \\
\hline Q1 & 925 & 179 & 1.0 & 1.0 & 1.0 & 1.0 \\
\hline Q2 & 848 & 153 & $0.96(0.77$ to 1.19$)$ & 0.94 (0.76 to 1.17$)$ & $0.93(0.74$ to 1.16$)$ & $0.92(0.74$ to 1.15$)$ \\
\hline Q3 & 637 & 103 & $1.00(0.79$ to 1.28$)$ & $1.02(0.80$ to 1.30$)$ & $1.00(0.78$ to 1.28$)$ & 0.96 (0.75 to 1.23$)$ \\
\hline Q4 & 731 & 146 & $1.37(1.10$ to 1.71$)$ & $1.36(1.09$ to 1.70$)$ & 1.31 (1.05 to 1.64$)$ & $1.27(1.01$ to 1.59$)$ \\
\hline Linear trend, $p$ value & & & 0.006 & 0.006 & 0.016 & 0.040 \\
\hline \multicolumn{7}{|l|}{ TV viewing* } \\
\hline Q1 & 793 & 125 & 1.0 & 1.0 & 1.0 & 1.0 \\
\hline Q2 & 837 & 140 & $1.01(0.80$ to 1.29$)$ & $0.98(0.77$ to 1.25$)$ & $0.94(0.74$ to 1.20$)$ & $0.93(0.73$ to 1.19$)$ \\
\hline Q3 & 812 & 179 & $1.28(1.02$ to 1.61$)$ & 1.21 (0.96 to 1.53$)$ & $1.15(0.91$ to 1.45$)$ & $1.13(0.90$ to 1.43$)$ \\
\hline Q4 & 699 & 140 & $1.12(0.88$ to 1.43$)$ & 1.07 (0.84 to 1.37$)$ & $1.02(0.80$ to 1.30$)$ & 0.99 (0.77 to 1.27$)$ \\
\hline Linear & & & 0.134 & 0.272 & 0.538 & 0.671 \\
\hline \multicolumn{7}{|l|}{ Total sedentary time* } \\
\hline Q1 & 895 & 146 & 1.0 & 1.0 & 1.0 & 1.0 \\
\hline Q2 & 687 & 116 & 0.99( & 0.95 & 0.92 & $0.92(C$ \\
\hline Q3 & 845 & 172 & to 1.56$)$ & o 1.50) & $1.14(0.92$ to 1.43$)$ & $1.12(0.89$ to 1.40$)$ \\
\hline Q4 & 714 & 147 & 1.34 (1.06 to 1.68$)$ & $1.30(1.03$ to 1.63$)$ & $1.22(0.97$ to 1.55$)$ & $1.20(0.95$ to 1.51$)$ \\
\hline Linear trend, $p$ value & & & 0.003 & 0.007 & 0.028 & 0.053 \\
\hline \multicolumn{7}{|l|}{ Cardiorespiratory fitnesst } \\
\hline Low & 1105 & 262 & 1. & 1. & 1.0 & 1.0 \\
\hline Middle & 1025 & 171 & $0.82)$ & $0.72(c$ & $0.73(0$ & 0.80 (0.65 to 0.99$)$ \\
\hline High & 1011 & 148 & 0.60 (0.49 to 0.73$)$ & $0.66(0.54$ to 0.80$)$ & 0.67 (0.55 to 0.82$)$ & 0.76 (0.59 to 0.97$)$ \\
\hline Linear trend, $p$ value & & & $<0.001$ & $<0.001$ & 0.030 & 0.030 \\
\hline
\end{tabular}

*Total sedentary time (ie, the sum of reported TV viewing and car time) was categorised into sample-specific quartiles (Q): Q1 (0-10 h/week), Q2 (11-15 h/week), Q3 (16-22 h/week) and Q4 ( $\geq 23 \mathrm{~h} /$ week). Quartiles of car time: (Q): Q1 (0-4 h/week), Q2 (5-7 h/week), Q3 (8-10 h/ week) and Q4 ( $\geq 11 \mathrm{~h} /$ week). Quartiles of TV viewing: (Q): Q1 (0-3 h/week), Q2 (4-7 h/week), Q3 (8-12 h/week) and Q4 ( $\geq 13 \mathrm{~h} /$ week). †Cardiorespiratory fitness was categorised into age (20-39, 40-49, 50-59 and $\geq 60$ years) and gender-specific tertiles based on the distribution of the sample.

¥Cox proportional hazard models were utilised to estimate the HR and $95 \%$ Cls.

§Adjusted for age and gender.

TAdjusted for age, gender, current smoking, alcohol, personal history of hypertension, personal history of diabetes and family history of CVD. ${ }^{*}$ Adjusted for age, gender, current smoking, alcohol, personal history of hypertension, personal history of diabetes, family history of CVD and cardiorespiratory fitness or sedentary time.

††Adjusted for age, gender, current smoking, alcohol, personal history of hypertension, personal history of diabetes, family history of CVD, cardiorespiratory fitness or sedentary time, physical activity, BMI, total cholesterol, systolic blood pressure and glucose.

BMI, body mass index; CVD, cardiovascular disease; PY, person years; Q, quartile; TV, television.

were at reduced mortality risk irrespective of sedentary time (table 3). For example, participants who were in the middle/high fitness strata and in the fourth quartile of sedentary time had a $40 \%$ decreased risk for mortality ( $\mathrm{HR}=0.60,95 \%$ CI 0.43 to 0.86$)$; whereas those in the middle/high fitness category and the lowest quartile of sedentary time were similarly at $40 \%$ reduced mortality risk $(\mathrm{HR}=0.60,95 \%$ CI 0.44 to 0.82$)$. In comparison, participants in the low fitness strata with lower levels of total sedentary time had a reduced risk for mortality; however, the association was statistically significant only among those classified in the second quartile of sedentary time (second quartile: $\mathrm{HR}=0.63,95 \%$ CI 0.44 to $0.90)$. A similar pattern was observed when examining the joint effects of car time and fitness on mortality. Specifically, participants in the low fitness strata who had lower levels of car time had reduced mortality risk (first quartile: $\mathrm{HR}=0.63,95 \%$ CI 0.46 to 0.88 ; second quartile: $\mathrm{HR}=0.61,95 \%$ CI 0.44 to 0.85 ); whereas those in the middle/high fitness group were at a similarly lower risk for mortality both in the lowest and highest levels of car time (first quartile: $\mathrm{HR}=0.58,95 \%$ CI 0.42 to 0.80 ; fourth quartile: $\mathrm{HR}=0.60,95 \%$ CI 0.42 to 0.86 ). With regard to the joint effects of TV viewing and fitness on mortality risk, few statistically significant findings were observed (see table 3 ).

\section{DISCUSSION}

The present study aimed to determine whether sedentary behaviour is associated with increased mortality risk irrespective of and alongside fitness among a cohort of adults. Study findings reveal a significant relationship between prolonged sedentary time and increased mortality risk in models not controlling for fitness. However, once fitness was taken into account the sedentary behaviour-mortality relationship was less pronounced. Specifically, being sedentary for 23 or more hours weekly significantly increased mortality risk by $29 \%$, while accounting for confounders with the exception of fitness. Once fitness was added into the model, then increased mortality risk from prolonged sedentary time 
Table 3 Joint effects of sedentary time and cardiorespiratory fitness on all-cause mortality

\begin{tabular}{|c|c|c|c|c|}
\hline & \multicolumn{4}{|c|}{ Cardiorespiratory fitness* } \\
\hline & \multicolumn{2}{|l|}{ Low } & \multicolumn{2}{|c|}{ Middle/high } \\
\hline & Cases & HRt $(95 \% \mathrm{Cl})$ & Cases & HR† (95\% Cl) \\
\hline \multicolumn{5}{|c|}{ Car time } \\
\hline Q1 & 67 & $0.63(0.46$ to 0.88$)$ & 112 & $0.58(0.42$ to 0.80$)$ \\
\hline Q2 & 62 & $0.61(0.44$ to 0.85$)$ & 91 & 0.52 (0.38 to 0.72$)$ \\
\hline Q3 & 49 & 0.71 (0.49 to 1.01$)$ & 54 & 0.51 (0.35 to 0.73$)$ \\
\hline Q4 & 84 & 1.0 & 62 & $0.60(0.42$ to 0.86$)$ \\
\hline \multicolumn{5}{|c|}{ TV viewing $\ddagger$} \\
\hline Q1 & 39 & $0.93(0.63$ to 1.38$)$ & 86 & $0.74(0.53$ to 1.04$)$ \\
\hline Q2 & 66 & $0.93(0.67$ to 1.30$)$ & 74 & 0.65 (0.46 to 0.92$)$ \\
\hline Q3 & 81 & $0.97(0.71$ to 1.34$)$ & 95 & 0.91 (0.66 to 1.27$)$ \\
\hline Q4 & 76 & 1.0 & 64 & 0.68 (0.48 to 0.97$)$ \\
\hline \multicolumn{5}{|c|}{ Total sedentary time $\ddagger$} \\
\hline Q1 & 48 & $0.71(0.50$ to 1.02$)$ & 98 & 0.60 (0.44 to 0.82$)$ \\
\hline Q2 & 47 & $0.63(0.44$ to 0.90$)$ & 69 & 0.58 (0.41 to 0.81$)$ \\
\hline Q3 & 80 & 0.80 (0.59 to 1.09$)$ & 92 & 0.68 (0.49 to 0.93$)$ \\
\hline Q4 & 87 & 1.0 & 60 & $0.60(0.43$ to 0.86$)$ \\
\hline \multicolumn{5}{|c|}{$\begin{array}{l}\text { *Cardiorespiratory fitness was categorised into age }(20-39,40-49,50-59 \text {, and } \geq 60 \text { years) and gender-specific tertiles based on the } \\
\text { distribution of the sample. Cardiorespiratory fitness was then dichotomised into low and middle/high for the joint effects analysis. } \\
\text { tCox proportional hazard regression was utilised to estimate the HR and } 95 \% \text { Cls. The model was adjusted for age, gender, current smoking, } \\
\text { alcohol, personal history of hypertension, personal history of diabetes, family history of CVD, physical activity, BMl, total cholesterol, systolic } \\
\text { blood pressure and glucose. } \\
\text { tTotal sedentary time (ie, the sum of reported TV viewing and car time) was categorised into sample-specific quartiles }(\mathrm{Q}) \text { : Q1 (0-10 h/week), } \\
\mathrm{Q} 2 \text { (11-15 h/week), Q3 (16-22 h/week) and Q4 ( } \geq 23 \mathrm{~h} / \text { week). Quartiles of car time: (Q): Q1 (0-4 h/week), Q2 (5-7 h/week), Q3 (8-10 h/ } \\
\text { week) and Q4 ( } \geq 11 \mathrm{~h} / \text { week). Quartiles of TV viewing: (Q): Q1 (0-3 h/week), Q2 (4-7 h/week), Q3 (8-12 h/week) and Q4 ( } \geq 13 \mathrm{~h} / \text { week). } \\
\text { BMI, body mass index; CVD, cardiovascular disease; Q, quartile; TV, television. }\end{array}$} \\
\hline
\end{tabular}

was $22 \%$. This $7 \%$ reduction in mortality risk likely stems from the protective health effects of fitness. ${ }^{14}$ Notably, when accounting for additional clinical variables (eg, BMI, blood pressure) that could be on the causal pathway between sedentary time and mortality, ${ }^{32}$ then mortality risk was reduced by an additional $2 \%$. This finding is understandable since studies have found that lower levels of sedentary behaviour have been linked to lower obesity and cardiometabolic risk which, in turn, could potentially lower morality risk. ${ }^{833} 34$ Thus, including these intermediate variables into the model is likely to confound the relationship between the exposure and outcome. $^{113235}$

In addition to examining the effects of total sedentary time on mortality, we also examined the relationship between time spent in a car and TV viewing in relation to mortality risk. Study results reveal the more time spent in a car significantly increased mortality risk even while taking fitness into account. This finding is consistent with a previous CCLS observing that longer commute distances are associated with elevated blood pressure even while considering the protective effects on both physical activity and fitness. ${ }^{17}$ Our null findings pertaining to TV viewing and mortality could potentially stem from: (1) the fact that while car time exclusively involves sitting, individuals watching TV could be multitasking (eg, moving about while watching TV viewing); or (2) the amount of time participants spent watching TV among this cohort is markedly lower than present day TV viewing habits. The later explanation might be more likely since most of the literature has observed higher mortality risk for those watching excessive amounts of TV. ${ }^{36}$

We additionally examined the relationship between fitness and mortality, finding that higher fitness levels reduced mortality risk irrespective of controlling for sedentary behaviour and the intermediate variables. This is indicative of the robust and causal relationship between fitness and mortality. ${ }^{14}$ Current findings pertaining to the protective effects of fitness (eg, $24 \%$ mortality reduction in the high fit strata fully adjusted model) are consistent with a large body of the literature that emphasises the importance of achieving higher fitness levels to obtain health benefits. ${ }^{14}$ Previous research has found $10-25 \%$ increased survival with a 1-MET increase in fitness. ${ }^{14}$ This represents a relatively small incremental change that is achievable for most individuals through increasing physical activity with the goal of reaching/ exceeding physical activity guidelines; that is, $150 \mathrm{~min}$ of moderate-intensity or $75 \mathrm{~min}$ of vigorous-intensity physical activity per week (or a combination of both). ${ }^{14} 29$ Thus, while decades of research emphasise the health benefits of increasing fitness levels, particularly for individuals with low levels of fitness, ${ }^{14}$ the evidence pertaining to sedentary behaviour and health outcomes (independent of physical activity) is accumulating but not as well established.

The most recent systematic review/meta-analysis on the topic conducted by Biswas et $a l^{11}$ found a $24 \%$ increased all-cause mortality risk for prolonged 
sedentary behaviour, when adjusting for physical activity; however, virtually all studies adjusted for self-reported physical activity, and none considered the protective impact of fitness. They additionally observed that high sedentary time coupled with low levels of physical activity resulted in an even higher risk $(46 \%)$ for all-cause mortality. ${ }^{11}$ An earlier study by Warren $e t a l^{19}$ found that prolonged sedentary behaviour increased cardiovascular disease mortality risk in a larger sample of men from the Cooper Clinic; however, they relied on self-reported physical activity, did not take fitness into account, and therefore did not comparatively examine its impact on mortality. Thus, in the current study, we demonstrate that sedentary behaviour is related to mortality risk, yet fitness 'buffers' some of the adverse health effects of total sedentary behaviour. The underlying mechanism as to why increased sedentary time leads to higher mortality risk warrants further investigation. The hypothesised biological mechanism of the unique impact of sedentary time, described elsewhere, ${ }^{37}$ includes the suppression of lipoprotein lipase activity, which results in the reduction of HDL-cholesterol and increased insulin resistance. ${ }^{12} 37{ }^{38}$ In a previous study of the CCLS cohort, we observed that sedentary time was cross-sectionally related to a proxy of insulin resistance even after adjusting for fitness. ${ }^{12}$

Current study findings should be tempered by the study's limitations. We examined a sample of adults who attended a preventive medicine clinic with objectively measure fitness and a multitude of information on patients' health. Thus, examination of the study question among a more representative sample is warranted to generalise findings. Further, while fitness was measured via maximal exercise testing, sedentary time was based on self-reported data on TV viewing and time spent in a car at baseline, which are proxies of sedentary behaviour and do not include all domains of sitting (eg, occupational sitting). Furthermore, participants' TV viewing habits and the car time measured at baseline (1982) are likely lower than present day sedentary behaviours. ${ }^{2}$ In addition, sedentary behaviours and physical activity were based on self-report, which might be subject to under-reporting or over-reporting. Moreover, the exposure measures (ie, sedentary behaviour, physical activity and fitness), assessed at baseline, might have changed during the follow-up period. ${ }^{18}$ Finally, dietary information was not available in the data set, and therefore was not adjusted for in the multivariable analysis. ${ }^{12}$

In summary, this is the first study to account for fitness when examining the sedentary behaviour-mortality relationship. Findings reveal that increased total sedentary time is related to higher mortality risk from all causes when fitness is not accounted for; however, once controlling for fitness the sedentary behaviour-mortality relationship is reduced. Fitness may also buffer some of the negative effects of time spent in a car; however, the inverse relationship between car time and mortality risk remained significant despite the inclusion of fitness into the models. Thus, higher levels of fitness appear to have some protective effects from prolonged sedentary time by lowering mortality risk. In addition, higher levels of fitness are protective against mortality risk irrespective of sedentary time. Therefore, increasing fitness levels through meeting or exceeding physical activity guidelines is of paramount public health importance. Nonetheless, additional research is needed to explore the relationship between sedentary behaviour and morbidity and mortality while taking the protective effects of fitness into account.

Acknowledgements The authors thank Dr Kenneth H Cooper MD, MPH for establishing the Cooper Center Longitudinal Study as well as the Cooper Clinic physicians and technicians for data collection. Additionally, they thank The Cooper Institute staff for data management, and the study participants. They are also grateful to John Daniel, MA of the American Cancer Society for editorial assistance.

Contributors All authors significantly contributed to the study to justify authorship. KS, CEF, CEB, KPG conceived the study. KS led the writing; CEF performed statistical analysis. All authors (KS, CEF, CEB, BTN, VYN, KPG) participated in interpreting the analyses and findings, and participated in the writing and/or editing/critically revising the paper.

Funding A portion of this work was supported by NIH AG09545.

Competing interests None declared.

Ethics approval The CCLS receives annual approval from the Cooper Institute Institutional Review Board and the present investigation received approval from the Committee for the Protection of Human Subjects at the University of Texas Health Science Center at Houston.

Provenance and peer review Not commissioned; externally peer reviewed.

Data sharing statement No additional data are available.

Open Access This is an Open Access article distributed in accordance with the Creative Commons Attribution Non Commercial (CC BY-NC 4.0) license, which permits others to distribute, remix, adapt, build upon this work noncommercially, and license their derivative works on different terms, provided the original work is properly cited and the use is non-commercial. See: http:// creativecommons.org/licenses/by-nc/4.0/

\section{REFERENCES}

1. Owen N, Sparling PB, Healy GN, et al. Sedentary behavior: emerging evidence for a new health risk. Mayo Clin Proc 2010;85:1138-41.

2. Matthews CE, Chen KY, Freedson PS, et al. Amount of time spent in sedentary behaviors in the United States, 2003-2004. Am J Epidemiol 2008;167:875-81.

3. Sedentary Behaviour Research Network. Letter to the editor: standardized use of the terms "sedentary" and "sedentary behaviours". Appl Physiol Nutr Metab 2012;37:540-2.

4. Shuval K, Gabriel KP, Leonard T. TV viewing and BMI by race/ ethnicity and socio-economic status. PLOS ONE 2013;8:e63579.

5. Healy GN, Matthews CE, Dunstan DW, et al. Sedentary time and cardio-metabolic biomarkers in US adults: NHANES 2003-06. Eur Heart J 2011;32:590-7.

6. Gardiner PA, Healy GN, Eakin EG, et al. Associations between television viewing time and overall sitting time with the metabolic syndrome in older men and women: the Australian Diabetes, Obesity and Lifestyle study. J Am Geriatr Soc 2011;59:788-96.

7. Thorp AA, Healy GN, Owen N, et al. Deleterious associations of sitting time and television viewing time with cardiometabolic risk biomarkers: Australian Diabetes, Obesity and Lifestyle (AusDiab) study 2004-2005. Diabetes Care 2010;33:327-34.

8. Veerman JL, Healy GN, Cobiac LJ, et al. Television viewing time and reduced life expectancy: a life table analysis. Br J Sports Med 2012;46:927-30.

9. Chau JY, Grunseit AC, Chey T, et al. Daily sitting time and all-cause mortality: a meta-analysis. PLOS ONE 2013;8:e80000. 
10. Ford ES, Caspersen CJ. Sedentary behaviour and cardiovascular disease: a review of prospective studies. Int $J$ Epidemiol 2012;41:1338-53.

11. Biswas A, Oh PI, Faulkner GE, et al. Sedentary time and its association with risk for disease incidence, mortality, and hospitalization in adults: a systematic review and meta-analysis. Ann Intern Med 2015;162:123-32.

12. Shuval K, Finley CE, Barlow CE, et al. Sedentary behavior, cardiorespiratory fitness, physical activity, and cardiometabolic risk in men: the cooper center longitudinal study. Mayo Clin Proc 2014;89:1052-62.

13. Sui X, LaMonte MJ, Laditka JN, et al. Cardiorespiratory fitness and adiposity as mortality predictors in older adults. J Am Med Assoc 2007;298:2507-16.

14. Kaminsky LA, Arena R, Beckie TM, et al. The importance of cardiorespiratory fitness in the United States: the need for a nationa registry: a policy statement from the American Heart Association. Circulation 2013;127:652-62.

15. Blair SN. Physical inactivity: the biggest public health problem of the 21st century. Br J Sports Med 2009;43:1-2.

16. Willis BL, Gao A, Leonard D, et al. Midlife fitness and the development of chronic conditions in later life. Arch Intern Med 2012;172:1333-40.

17. Hoehner CM, Barlow CE, Allen P, et al. Commuting distance, cardiorespiratory fitness, and metabolic risk. Am J Prev Med 2012:42:571-8.

18. Shuval K, Barlow CE, Chartier KG, et al. Cardiorespiratory fitness, alcohol, and mortality in men: the Cooper Center longitudinal study. Am J Prev Med 2012;42:460-7.

19. Warren TY, Barry V, Hooker SP, et al. Sedentary behaviors increase risk of cardiovascular disease mortality in men. Med Sci Sports Exerc 2010;42:879-85.

20. Shuval K, Finley CE, Chartier KG, et al. Cardiorespiratory fitness, alcohol intake, and metabolic syndrome incidence in men. Med Sci Sport Exerc 2013;44:2125-31.

21. Pollock ML, Bohannon RL, Cooper $\mathrm{KH}$, et al. A comparative analysis of four protocols for maximal treadmill stress testing. Am Heart $J$ 1976;92:39-46.

22. Pollock ML, Foster C, Schmidt D, et al. Comparative analysis of physiologic responses to three different maximal graded exercise test protocols in healthy women. Am Heart J 1982;103:363-73.

23. Kendzor DE, Finley CE, Barlow CE, et al. The asssociation of fitness with reduced cardiometabolic risk among smokers. Am J Prev Med 2015;48:561-9.
24. Stampfer MJ, Willett WC, Speizer FE, et al. Test of the National Death Index. Am J Epidemiol 1984;119:837-9.

25. Farrell SW, Fitzgerald SJ, McAuley PA, et al. Cardiorespiratory fitness, adiposity, and all-cause mortality in women. Med Sci Sports Exerc 2010;42:2006-12.

26. Mukamal KJ, Chen CM, Rao SR, et al. Alcohol consumption and cardiovascular mortality among U.S. adults, 1987 to 2002. J Am Coll Cardiol 2010;55:1328-35

27. Barlow CE, Shuval K, Balasubramanian B, et al. Sitting time, physical activity and cardiorespiratory fitness: CCLS cohort. J Phys Act Health 2015.

28. Ainsworth BE, Haskell WL, Herrmann SD, et al. 2011 Compendium of physical activities: a second update of codes and MET values. Med Sci Sports Exerc 2011;43:1575-81.

29. US Department of Health and Human Services. Physical Activity Guidelines for Americans. 2008. http://www.health.gov/paguidelines/ pdf/paguide.pdf

30. Shuval K, Chiu CY, Barlow CE, et al. Family history of chronic disease and meeting public health guidelines for physical activity: the cooper center longitudinal study. Mayo Clin Proc 2013;88:588-92.

31. Lin DY, Wei LJ, Ying Z. Checking the cox model with cumulative sums of martingale-based residuals. Biometrika 1993;80:557.

32. Matthews CE, George SM, Moore SC, et al. Amount of time spent in sedentary behaviors and cause-specific mortality in US adults. Am J Clin Nutr 2012:95:437-45.

33. Healy GN, Dunstan DW, Salmon J, et al. Breaks in sedentary time: beneficial associations with metabolic risk. Diabetes Care 2008;31:661-6.

34. Dunstan DW, Kingwell BA, Larsen R, et al. Breaking up prolonged sitting reduces postprandial glucose and insulin responses. Diabetes Care 2012;35:976-83.

35. Rothman KJ, Greenland S, Lash TL. Modern epidemiology. 3rd edn. Philadelphia, PA: Lippincott Williams \& Wilkins, 2008.

36. Grøntved A, Hu FB. Television viewing and risk of type 2 diabetes, cardiovascular disease, and all-cause mortality: a meta-analysis. J Am Med Assoc 2011;305:2448-55.

37. Bey L, Hamilton MT. Suppression of skeletal muscle lipoprotein lipase activity during physical inactivity: a molecular reason to maintain daily low-intensity activity. J Physiol (Lond) 2003;551:673-82.

38. Hamilton MT, Hamilton DG, Zderic TW. Role of low energy expenditure and sitting in obesity, metabolic syndrome, type 2 diabetes, and cardiovascular disease. Diabetes 2007;56:2655-67. 